\title{
SKELETAL PATHOLOGICAL CONDITIONS OF LITHUANIAN MUMMIES
}

Dario Piombino-Mascali ${ }^{1}$, Justina Kozakaité ${ }^{1}$, Algirdas Tamošiūnas ${ }^{1}$, Ramūnas Valančiuss ${ }^{1}$, StePhanie PANZER ${ }^{2,3}$, Rimantas Jankauskas ${ }^{1}$

${ }^{1}$ Faculty of Medicine, Vilnius University, Vilnius, Lithuania

${ }^{2}$ Trauma Center Murnau, Murnau, Germany

${ }^{3}$ Paracelsus Medical University, Salzburg, Austria

\begin{abstract}
This report examines the bone lesions found during CT investigations of mummies from the Dominican Church of the Holy Spirit in Vilnius, Lithuania. Observed pathological conditions include a single fracture, osteoarthritis, malpositions and dental disease, as well as some normal variants of the human skeleton. These palaeopathological data provide a clearer picture of the mummies' osteobiographies, and serve as a reminder about the importance of noninvasive techniques for the investigation of historical preserved remains.
\end{abstract}

Keywords: mummies, bone, imaging, palaeopathology

\section{INTRODUCTION}

The Dominican Church of the Holy Spirit of Vilnius overlies a number of subterranean chambers which hold mummified and skeletonised human remains of clergymen and laypeople, as well as wooden coffins and precious textiles likely dating from the $18^{\text {th }} / 19^{\text {th }}$ centuries $\mathrm{AD}$ (5). This form of burial was typical of middle and upper-class members of the society, and is well-documented in Modern Europe (3). The mummies concerned are, in all likelihood, the results of environmental conditions conducive to the mummification process (7). A low average temperature, protection from rain and humidity by the overlying church and the wooden coffins, and a constant air flow may all have contributed 
to the preservation of these remains (1). In addition, preservation may have been aided by the pine-wood material some of the coffins were constructed from, which produces turpenoids with significant antifungal and antimicrobial properties. This would hinder the decomposers while environmental dehydration was occurring (6).

An early attempt to sort out the cellars and their content was carried out during the 1930s by students of the local University; however, the first report on those mummies was only produced in 1963, by forensic scientist Juozas Markulis (1913-1987). The accumulation of remains in one single cellar and changes in ventilation due to the installation of a glass resulted in the decomposition of most mummies, which unfortunately left few specimens to be retrieved in the current century. In 2011, within the framework of the "Lithuanian Mummy Project" (a documentation, study and conservation of this historic material) some bodies remained in such a remarkable state of preservation to allow for a non-invasive study to be performed, and as such were CT-scanned. While soft tissue findings will be discussed elsewhere, this paper reports on the bone lesions that were observed on five subjects during the imaging study.

\section{MATERIALS AND METHODS}

Seven human mummies of both sexes and different age classes were selected for this study due to their good or remarkable external preservation status. These remains were examined at the Central Branch of Vilnius University Hospital using a Philips Mx8000 Dual CT scanner (Best, the Netherlands). All examinations were obtained as whole-body CT scans in helical technique with a slice thickness of $1.3 \mathrm{~mm}$, an increment of $1.3 \mathrm{~mm}$, a tube voltage of $120 \mathrm{kV}$, and a tube current of $90 \mathrm{~mA}$. Palaeoradiological evaluations as well as the performance of different reconstructions were carried out at the Picture Archiving and Communicating System (ImpaxEE, Agfa Health-Care, Bonn, Germany) and the CT adjacent workstation (ADW 4.3, General Electrics, Milwaukee, USA) in the Department of Radiology, Trauma Center Murnau, Germany. Pathological conditions were observed on six individuals. One case of a child mummy with rickets and hydrocephalus has already been described elsewhere (12).

\section{RESULTS}

\section{Skeletal Preservation}

Preservation of the skeletal system in the investigated mummies varied from complete (affected only by some dislocations) to incomplete due to missing 
elements. The three complete subjects were affected by postmortem dislocations involving in one case some teeth, the cranio-cervical junction, the atlas, and the sterno-costal joints; some teeth, the second to fifth lumbar vertebrae, and the sacrum in another; and the temporomandibular joint, teeth of the right mandible, cranio-cervical junction, and atlas in the last. The other three subjects showed dislocations and missing parts: one individual displayed dislocation of the cranio-cervical junction, the first and second cervical vertebrae, and some dislocated teeth, as well as missing teeth and feet; another subject showed dislocation of the cranio-cervical elements and atlas, most parts of the thoracic and lumbar spine and sacrum as well as the ribs; fragments of vertebrae were noted intracranially, together with brain remnants; missing parts included portions of the thoracic and lumbar spine and parts of the visible vertebral bodies (especially loss of central vertebral parts). Finally, the last skeleton was missing the left foot distal to the talus.

\section{Palaeopathology}

The main condition encountered during the study of this small mummy sample was degenerative joint disease (osteoarthritis).

The skeleton of VD3, an obese adult male, showed slight arthritis of the cervical and lumbar spine and moderate arthritis of the thoracic spine (Fig. 1a), slight arthritis of the iliosacral joints (left more so than right) and the symphysis. Moderate arthritis was observed in both knees; the second case, an obese adult female (VD9) showed a cervical spine with slight arthritis, a thoracic spine with marked right convex scoliosis with stabilising spondylophytes on the left side and partial osseous bridging (Fig. 2a), as well as arthritis of the posterior facets, the costovertebral, and the costotransversal joints. Ossification between the spinal processes was also observed, and asymmetry of the thorax was apparent. Arthritis also affected the sterno-clavicular joints, which was more pronounced on the right side (Fig. 2b), and the manubrio-sternal joint. Lumbar spine showed slight arthritis, the iliosacral joints some slight arthritis, and the hips some moderate arthritis, again, more advanced on the right side (Fig. 2c). Also the symphysis showed slight to moderate arthritis and it was possible to note generalised enthesopathies of the pelvis. Finally, bilateral gonarthritis was diagnosed in the lateral femoro-tibial and femoro-patellar compartments laterally, yet more pronounced on the right side (Fig. 2d). The skeleton of VD12, an adult male, showed slight arthritis of the cervical spine, as well as slight to moderate arthritis of the thoracic spine. Furthermore, beyond the abovementioned scoliosis, another malposition was seen in one case, VD10, an obese young adult female who showed bilateral hallux valgus (Fig. 3). Moreover, an 
interesting finding on VD12 was that of a herniation pit on the right proximal femur (Fig. 4a), which seems to have been common in the past as well as today.

Trauma was only present in one case, VD3, with a fracture of the fourth rib on the right side with little formation of callus and marginal osseous bridging (Fig. 1b).

Additionally, a case of benign tumour (namely a haemangioma in the fifth lumbar vertebra of VD9) (Fig. 2e) was also observed.

Finally, dental pathologies involved three of these subjects, namely VD9, VD12, and VD14. The first case showed abscesses/granulomas on two teeth as well as a missing tooth with atrophy of the alveolar crest/ridge, indicating antemortem loss; the second showed an abscess/granuloma, as well as antemortem loss for four teeth; and the third case showed abscesses/granulomas affecting three teeth.
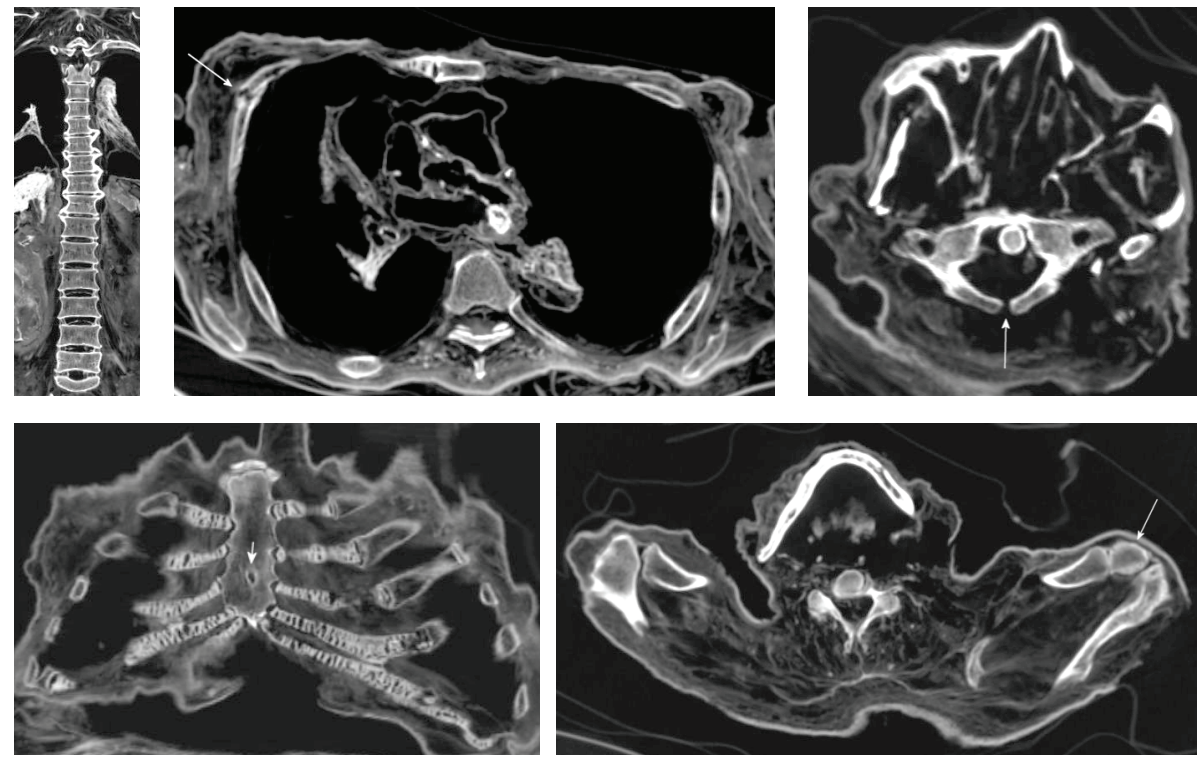

Figure 1. Case VD3.

a. Coronal reformation CT image showing moderate arthritis of the thoracic spine in the form of spondylophytes and narrowing of the disc spaces, and slight arthritis of the lumbar spine.

b. Axial reformation CT image revealing a fracture of the fourth rib on the right side with little formation of callus and marginal osseous bridging (arrow).

c. Axial reformation CT image illustrating fissured vertebral arch of the atlas (arrow).

d. Coronal reformation CT image revealing a hole in the corpus sterni (arrow). Note distinct calcification of rib cartilages.

e. Axial reformation CT image showing an os acromiale on the left side (arrow). For comparison normal shoulder anatomy is shown on the right side. 

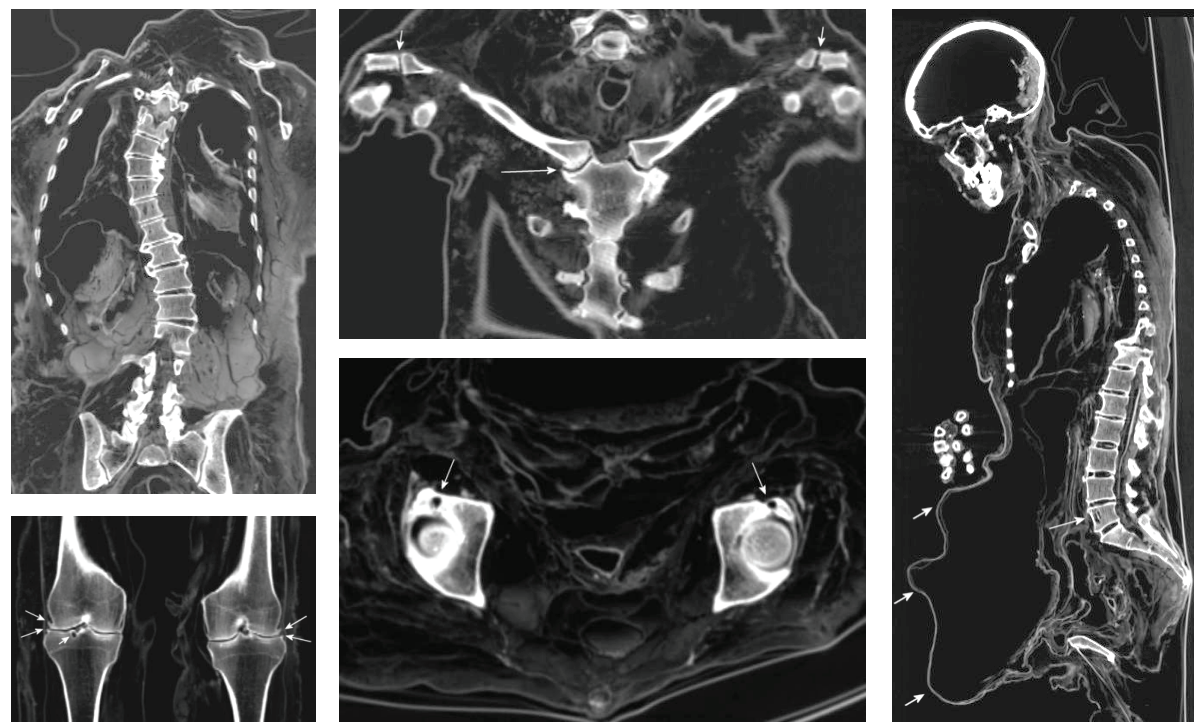

Figure 2. Case VD9.

a. Coronal reformation CT image illustrating marked S-shaped thoracic spine with stabilising spondylophytes and partial osseous bridging.

b. Coronal reformation CT image showing arthritis of the sternoclavicular joints, more pronounced on the right side in the form of distinct osteophytes (long arrow). Note slight arthritis of both acromioclavicular joints in the form of partially-irregular bearing area (short arrows).

c. Axial reformation CT image revealing subchondral cysts in the acetabulum of both hip joints (arrows).

d. Coronal reformation CT image showing arthritis in both knees, with visible marginal osteophytes in the lateral femorotibial compartment (long arrows). Note additional subchondral cyst in the right tibia (short arrow).

e. Sagittal reformation CT image depicting an intraosseous haemangioma in the fifth lumbar vertebra (long arrow). Note the distinct pendulous abdomen overhanging the pelvic region and upper thighs (short arrows).

\section{Anatomical variations}

Anatomical variations observed during the study include mummy VD3 showing an anomaly of closure of the posterior arch of the atlas (spina bifida) (Fig. 1c), a hole in the body of the sternum (fissura sterni congenita) (Fig. 1d), and an os acromiale on the left side (Fig. 1e). Mummy VD12 has a slight asymmetry of the thorax with reduced volume of the left apical part; and mummy VD14 with the sacrum displaying a slight anatomical variation: incomplete fusion of both neuroforamina of the third sacral vertebra with adjacent sclerosis, which can also represent a sacro-caudal transitional vertebra (Fig. 5). 


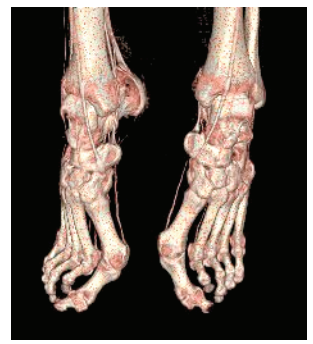

Figure 3. Three-dimensional reformation CT image of the feet of case VD10 illustrating bilateral hallux valgus. Note preservation of tendons.
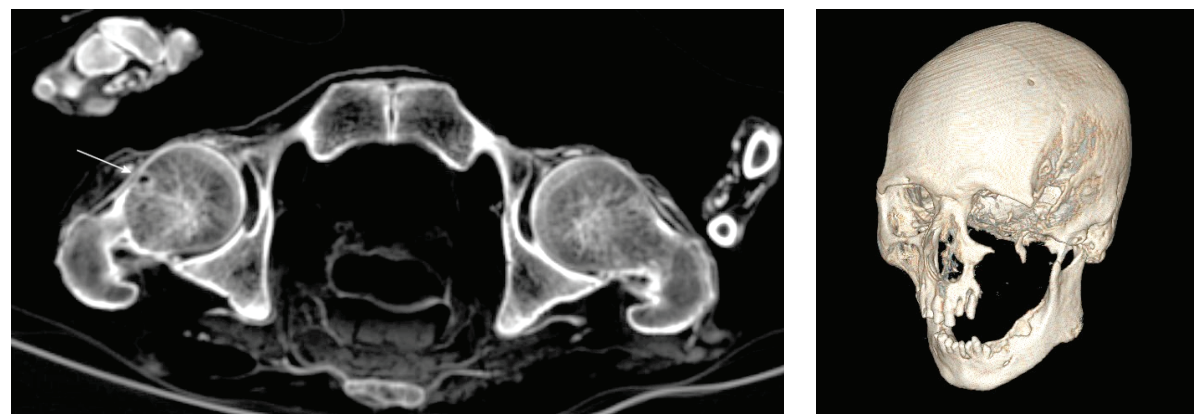

Figure 4. Case VD12.

a. Axial reformation CT image revealing a herniation pit at the anterior aspect of the right proximal femur (arrow).

b. Three-dimensional reformation CT image of the head showing a large osseous defect of the left mid-face.

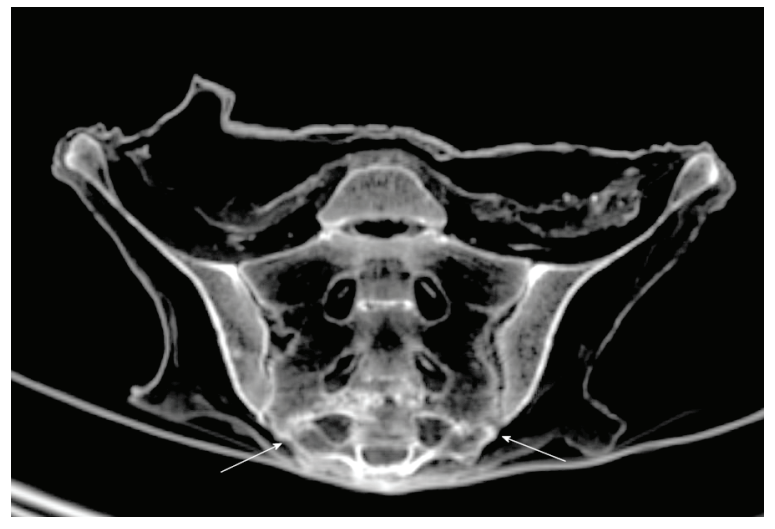

Figure 5. Coronal reformation CT image of the sacrum of case VD14 showing incomplete fusion of both neuroforamina of the third sacral vertebra with adjacent sclerosis (arrows), which can also represent a sacro-caudal transitional vertebra.

\section{DISCUSSION}

Despite being aware of some limitations, including the representativeness and paucity of the sample, the CT investigation helped to identify some pathological conditions which are useful for a thorough understanding of the mummies' 
osteobiographies. The most common palaeopathological finding was definitely osteoarthritis, affecting three of the subjects concerned. Such a condition is characterised by the loss of joint cartilage and subsequent lesions resulting from interosseous contact within diarthrodial joints, and is usually visible during the fourth decade of life. It is age-progressive and with no gender predilection. While several factors can determine its onset, one of the causes is certainly obesity, which is obvious in two of the mummies affected (2). Clinical data indicate that $45 \%$ of people suffering from gonarthitis are in fact obese. With increasing weight, the risk for gonarthitis and functional severity escalates. Obesity precedes the disease, leading to mechanical stress and articular strains (4). As mentioned before, one male mummy showed mild changes of the knee, while another showed clearer evidence of the condition.

In addition, the case displaying a herniation pit is worth mentioning as these entities, which appear as round to oval radioluciencies in the proximal superior quadrant of the femoral neck, seem to play a part in femoroacetabular impingement of the hip joint which is known to cause osteoarthritis (11).

As far as injuries are concerned, the only evidence was provided by a healed rib fracture. This type of fracture is generally attributed to direct or indirect blunt chest traumas. However, as the subject concerned suffered from pulmonary tuberculosis (Piombino-Mascali et al., original data), an association between this fracture and the presence of a respiratory disease accompanied by severe and prolonged coughing should also be considered (9).

Our mummy sample also revealed the presence of malpositions, such as scoliosis and hallux valgus deformity. The former consists of a lateral curvature of the spine with rotation of the vertebrae, in most cases (80\%) idiopathic in origin. Population studies have shown that this is a familial disease in $30 \%$ of cases (2). However, not many true cases have been described in the palaeopathological literature due to lack of optimal preservation of the vertebral column. Because of stabilising osteophytes on the left side, this case of scoliosis should be considered an antemortem change, not associated with possible tuberculosis due to the fact that fibrotic changes are not so distinct. The latter, instead, is the abnormal deviation of the great toe, which in palaeopopulations is commonly associated to the use of specific styles of footwear (8).

Another interesting finding is also that of a haemangioma, a benign, solitary and small tumour of proliferating blood vessels and vascular sinusoids mostly affecting older females (2). In the skeleton, this formation is commonly found in the lower spine, and in severe forms it can mimic the signs of Pott's disease, metastatic carcinoma or Paget's disease. Advanced stages of this condition 
would result in serious back pain, radicular pain or even spinal cord compression. Probable cases of such a condition have been occasionally described in the palaeopathological literature (1).

Finally, the presence of periapical abscesses/granulomas, which are spheres of soft tissue lesions surrounding the tooth apex and creating a space, should be mentioned. These lesions basically indicate that tooth was non-vital at the time of death, and in general, a poor dental hygiene among the investigated group (10).

\section{Taphonomy}

Post-depositional taphonomic changes were also noted, and include a defect of the left frontal bone of VD3, measuring up to $6.5 \mathrm{~cm}$, with sharp edges, bony fragments inside the skull and the galea hanging through the defect into the skull. There is also a huge osseous defect on VD12, of the left midface affecting the lateral, medial and inferior margin of the orbit, the lower parts of the ethmoidal cells, the margin of the middle cranial fossa, parts of the sphenoid bone, the zygomatic arch, parts of the pterygoid process, complete margins of the maxillary sinus, the maxilla dorsal to D3, and parts of the bony palate (Fig. $4 \mathrm{~b})$. However, the margins of the defect are not completely sharp and no bony fragments or preserved soft tissues were seen inside the defect, indicating that further work is needed to clarify its origin. If a tumour is to be excluded, the most probable cause of this defect should be taphonomic, including postmortem trauma.

\section{CONCLUSIONS}

The CT investigation of the Vilnius mummies has proved useful for assessing the presence of bone pathologies which would not have been seen otherwise, due to the presence of soft tissues covering the bony elements. Despite limitations, the study offers a deeper picture of the Holy Spirit community, emphasising the role of non-invasive studies as the main method of analysing preserved remains.

\section{REFERENCES}

1. Aufderheide A.C. (2003). The Scientific Study of Mummies. Cambridge University Press. Cambridge.

2. Aufderheide A.C., Rodríguez Martín C. (1998). The Cambridge Encyclopedia of Human Paleopathology. Cambridge University Press. Cambridge. 
3. Frick D. (2013). Kith, Kin \& Neighbors. Communities \& Confessions in Seventeenth-Century Wilno. Corner University Press. Ithaca and London.

4. Glimet T., Massé J.P., Kuntz D. (1990). Obesity and gonarthitis. Revue du Rhumatisme et des Maladies Ostéo-articulaires, 53(7), 207-209.

5. Jankauskas R., Piombino-Mascali D. (2012). The Lithuanian Mummy Project: bioanthropological and paleopathological investigation of the human remains found in the Holy Spirit Dominican Church, Vilnius. Paleopath Newsl, 159, 12.

6. Knobloch K., Pauli A., Iberl B., Weigand H., Weis N. (1989). Antibacterial and antifungal properties of oil components. J Essent Oil Res, 1(3), 119-128.

7. Lynnerup N. (2007). Mummies. Yrbk Phys Anthropol, 45, 162-190.

8. Mafart B. (2007). Hallux valgus in a historical French population: paleopathological study of 605 first metatarsal bones. Joint Bone Spine, 74, 166-170.

9. Matos V. (2009). Broken ribs: paleopathological analysis of costal fractures in the human identified skeletal collection from the Museu Bocage, Lisbon, Portugal (Late $19^{\text {th }}$ to Middle $20^{\text {th }}$ centuries). Am J Phys Anthropol, 140, 25-38.

10. Ogden A. (2008). Advances in the palaeopathology of teeth and jaws. In: Pinhasi R., Mays S. (eds), Advances in Human Palaeopathology. Wiley. Chichester, 283-307.

11. Panzer S., Piombino-Mascali D., Zink A.R. (2012). Herniation pits in human mummies: A CT investigation in the Capuchin Catacombs of Palermo, Sicily. PLoS ONE 7(5), e36537.

12. Panzer S., Tamošiūnas A.,Valančius R., Jankauskas R., Piombino-Mascali D. (2013). Radiological evidence of rickets in a Lithuanian child mummy. RöFo, 185(7), 670-672.

\section{Address for correspondence:}

Dr Dario Piombino-Mascali

Department of Anatomy, Histology and Anthropology

Faculty of Medicine, Vilnius University

MK Čiurlionis street 21, LT-03101, Vilnius, Lithuania

E-mail: dario.piombino@mf.vu.lt 